\title{
Pesquisa e produção científica em economia da saúde no Brasil*
}

\author{
Eli lola Gurgel Andrade, Francisco de Assis Acúrcio, \\ Mariangela Leal Cherchiglia, Soraya Almeida Belisário, \\ Augusto Afonso Guerra Júnior, Daniele Araújo Campos \\ Szuster, Daniel Resende Faleiros, Hugo Vocurca Teixeira, \\ Grazzielle Dias da Silva, Thiago Santos Taveira**
}

SuMÁRIO： 1. Introdução; 2. Metodologia; 3. Resultados; 4. Conclusões.

SumMARY : 1. Introduction; 2. Methodology; 3. Results; 4. Conclusions.

\begin{abstract}
* Artigo recebido em abr. 2005 e aceito em nov. 2006.
** Eli Iola Gurgel Andrade é doutora em demografia e professora adjunta do Departamento de Medicina Preventiva e Social da Universidade Federal de Minas Gerais (UFMG). Endereço: Av. Alfredo Balena, 190, sala 9014 — CEP 30410-180, Belo Horizonte, MG, Brasil. E-mail:iola@medi cina.ufmg.br.

Francisco de Assis Acúrcio é pós-doutor em economia da saúde e professor associado do Departamento de Farmácia Social da UFMG. Endereço: Av. Antônio Carlos, 6627, sala 1048-B2 CEP 31270-901, Belo Horizonte, MG, Brasil. E-mail: fracurcio@gmail.com.

Mariangela Leal Cherchiglia é doutora em saúde pública e professora adjunta do Departamento de Medicina Preventiva e Social da UFMG. Endereço: Av. Alfredo Balena, 190, sala 9014 CEP 30410-180, Belo Horizonte, MG, Brasil. E-mail: cherchml@medicina.ufmg.br.

Soraya Almeida Belisário é doutora em saúde pública e professora adjunta do Departamento de Medicina Preventiva e Social da UFMG. Endereço: Av. Alfredo Balena, 190, sala 9014 - CEP 30410-180, Belo Horizonte, MG, Brasil. E-mail: dadaya@medicina.ufmg.br.

Augusto Afonso Guerra Júnior é doutorando em saúde pública e assessor técnico especializado do Departamento de Economia da Saúde, da Secretaria de Ciência, Tecnologia e Insumos Estratégicos do Ministério da Saúde. Endereço: Av. Alfredo Balena, 190, sala 9014 - CEP 30410180, Belo Horizonte, MG, Brasil. E-mail: augustoguerramg@gmail.com.

Daniele Araújo Campos Szuster é administradora e assessora técnica especializada do Departamento de Economia da Saúde, da Secretaria de Ciência, Tecnologia e Insumos Estratégicos do Ministério da Saúde. Endereço: Av. Alfredo Balena, 190, sala 9014 - CEP 30410-180, Belo Horizonte, MG, Brasil. E-mail: danieleacampos@gmail.com.

Daniel Resende Faleiros é engenheiro civil, bacharel em direito e assessor técnico especializado do Departamento de Economia da Saúde, da Secretaria de Ciência, Tecnologia e Insumos Estratégicos do Ministério da Saúde. Endereço: Av. Alfredo Balena, 190, sala 9014 - CEP 30410180, Belo Horizonte, MG, Brasil. E-mail: dfalleiros@gmail.com.
\end{abstract}


Palavras-chave: economia da saúde; produção científica; grupos de pesquisa; inquérito.

KEY WORDS: health economics; scientific production; research groups; survey.

Este artigo elabora um diagnóstico sobre a área de economia da saúde (ES) no Brasil, a partir de inquérito enfocando os grupos de pesquisa em ES cadastrados no Conselho Nacional de Desenvolvimento Científico e Tecnológico (CNPq) e de um levantamento da produção científica brasileira em ES, publicado entre janeiro de 1999 e junho de 2004 e disponibilizado na Biblioteca Virtual em Saúde Bireme (Paho/WHO). Os dados foram descritos considerando regiões geográficas, tipos de instituições e áreas temáticas de pesquisa. Dos grupos de pesquisa com trabalhos relacionados às áreas temáticas em ES, 48 apresentam ampla diversidade de atuação e concentram-se na região Sudeste do país. Apenas 14\% (376) das 2.617 publicações avaliadas se relacionam à ES. A maioria dos estudos foi publicada em 2002 e apresenta como principais temas: gestão, financiamento, eficiência alocativa e eqüidade na distribuição de recursos de saúde. A análise permite identificar aspectos importantes para compreender o desenvolvimento do campo da ES no Brasil, entre 1999 e 2004.

\section{Research and scientific production in health economics in Brazil} This article presents a diagnosis of the health economics (HE) in Brazil, based on a survey of HE research groups currently registered in the National Council for Scientific and Technological Development (CNPq), as well as an inventory of the Brazilian scientific production in HE, published between January 1999 and June 2004, available in the Bireme Virtual Health Library (Paho/WHO). It describes the data considering geographic regions, types of institution and topics of research. Of the research groups with works

Hugo Vocurca Teixeira é mestrando em economia da saúde e secretário adjunto de educação da Secretaria de Educação da Prefeitura de Belo Horizonte. Endereço: Rua Barbosa Resende, 16, ap. 201H CEP 30430-680, Belo Horizonte, MG, Brasil. E-mail: vocurca@yahoo.com.br.

Grazzielle Dias da Silva é mestranda em saúde pública e pesquisadora do Departamento de Medicina Preventiva e Social da UFMG. Endereço: Av. Alfredo Balena, 190, sala 9014 - CEP 30410-180, Belo Horizonte, MG, Brasil. E-mail: grazidiassilva@hotmail.com.

Thiago Santos Taveira é acadêmico de medicina da UFMG. Endereço: Av. Alfredo Balena, 190, sala 9014 - CEP 30410-180, Belo Horizonte, MG, Brasil. E-mail: taveira@medicina.ufmg.br. 
related to HE subject matters, 48 have very diversified activities, concentrated in the Southeastern region of the country. Only 14\% (376) of the 2.617 publications that had been evaluated were related to HE. Most of the studies were published in 2002 and their main topics were management, financing, allocative efficiency and equity in the distribution of health resources. The diagnosis allows the identification of important aspects that help understand the development of the HE field in Brazil between 1999 and 2004.

\section{Introdução}

A criação do Sistema Único de Saúde (SUS), nos anos 1980, ao expandir o direito aos serviços de saúde de forma integral e equânime, foi na contramão do discurso da reforma do Estado e da focalização das políticas sociais. Configurouse, portanto, no Brasil, um movimento inverso ao ocorrido nos demais países latino-americanos, no que tange às políticas públicas de saúde.

A demanda por mais eficiência e maior eficácia da ação governamental na gestão do sistema de saúde, visando a implementação dos princípios e diretrizes do SUS, estabelece a necessidade e a urgência do desenvolvimento e consolidação do campo da economia da saúde no país, no sentido de produzir estudos e pesquisas que contribuam para a consecução de tais finalidades. É essencial que as alternativas de alocação de recursos sejam analisadas no contexto da universalidade e integralidade e, dado o caráter econômico de bem público da assistência à saúde, é possível e necessário combinar eficiência e eqüidade em seu provimento.

Outro desafio é o processo de descentralização da gestão do SUS. A contradição entre a execução descentralizada das ações de saúde e a manutenção de recursos financeiros centralizados sob a gestão da União gera constantemente conflitos distributivos. A ES pode nesse ponto ser acionada para auxiliar a construção de propostas de alocação mais equânime de recursos e estudar contrapartidas financeiras das outras esferas de governo.

A ampliação da eficiência do sistema de saúde brasileiro no que se refere à provisão de serviços é de grande importância, tanto pelo lado da demanda, por meio da identificação das necessidades da população quanto pelo lado da oferta, em termos da forma como esses serviços são disponibilizados.

Além disso, a incorporação de tecnologia no setor saúde tem reflexos conhecidos sobre o custo do sistema, além de exigir mecanismos de regulação 
cada vez mais complexos e sofisticados. Logo, estudar o sistema de regulação e a incorporação de novas tecnologias é uma necessidade cada vez mais premente no sistema de saúde brasileiro.

Saes (2000) considera a incorporação da economia da saúde um auxílio indispensável na determinação das prioridades da gestão em saúde, pois propicia metodologias e/ou instrumentos gerenciais de avaliação econômica, contemplando estudos de oferta e demanda em saúde; estudos relativos à saúde suplementar e à organização dos provedores de serviços; avaliação de tecnologias médica e diagnóstica; análise dos sistemas de saúde, regulação e competição nesse mercado e outras possibilidades.

Para Medeiros (1999), a escassez de recursos destinados à saúde exige decisões alocativas que consistem em selecionar os beneficiários do sistema público de saúde e quais serviços serão oferecidos. A responsabilidade dessas decisões, segundo o autor, é imensa em um país como o Brasil, onde há grande demanda por saúde e impossibilidade de ampla parcela da população obter serviços fora do sistema público.

Pode-se dizer que atualmente a contribuição da economia para a esfera da assistência à saúde é bem conhecida e deriva da natureza dos bens e serviços oferecidos pelo sistema de atenção médica, assim como da estrutura dos mercados a eles relacionados. Em primeiro lugar, muitos desses bens e serviços podem ser classificados, segundo a teoria econômica, de "bens públicos". Um exemplo de bem público, aí, seria um programa para a erradicação de doenças infecto-contagiosas. A teoria econômica fundamenta uma importante justificativa para a intervenção governamental na produção desses bens. Em segundo lugar, há bens e serviços oferecidos pelo sistema de atenção médica que geram externalidades positivas, como os programas de vacinação. A teoria econômica indica que a presença de externalidades é uma condição para "falhas de mercado", uma situação na qual o mercado tende a prover um nível subótimo de bens e serviços. Em terceiro lugar, existem assimetrias de informação nos mercados de provisão de bens e serviços de saúde. Tais assimetrias implicam um risco substancial de "falhas de mercado", onde a regulação governamental passa a ser essencial.

Casasnovas e colaboradores (2003), ao enfocarem a atuação pública, observam que a maior parte dela se relaciona com atividades sociais (provisão de bens e serviços como educação, saúde, cultura, habitação ou bem-estar comunitário) e com atividades econômicas (infra-estruturas e comunicações, subvenções a setores produtivos), além do serviço da dívida. Os autores 
enfatizam que, para atuações de "bem público" a partir do ponto de vista da análise econômica, a exigência não pode ser outra do que a da própria avaliação econômica, ou seja, assegurar a transparência da decisão em termos de custos e benefícios sociais, a melhor observância de medidas, garantias procedimentais etc. (Casasnovas et al., 2003:16).

No campo científico, a economia da saúde se desenvolve a partir de estudos e pesquisas sistemáticas e da aplicação de instrumentos econômicos como ferramentas estratégicas e/ou operacionais do setor saúde. É imperativo ressaltar o importante papel das condições de vida das populações e suas conseqüências sobre a saúde para o estudo da dinâmica do mercado dos serviços dessa área. Del Nero (2002:19) define economia da saúde como "a aplicação do conhecimento econômico ao campo das ciências da saúde, em particular como elemento contributivo à administração dos serviços de saúde" ou, ainda,

ramo do conhecimento que tem por objetivo a otimização das ações de saúde, ou seja, o estudo das condições ótimas de distribuição dos recursos disponíveis para assegurar à população a melhor assistência à saúde e o melhor estado de saúde possível, tendo em conta meios e recursos limitados.

A ES apresenta-se atualmente como um campo multidisciplinar de produção científica e ação governamental bastante desenvolvido na Europa, Canadá, Estados Unidos e Austrália, reconhecendo-se que seu conhecimento é essencial para quem trabalha no planejamento, administração e gestão de serviços de saúde. Mesmo com a presença de estudos internacionais na área anteriores aos anos 1950, pode-se dizer que no Brasil a economia da saúde possui um desenvolvimento recente. Pode-se tomar a criação da Associação Brasileira de Economia da Saúde (Abres), em 1989, como a base de sua instituição no país. A produção científica na área vem, desde 1993, se institucionalizando, principalmente por meio do apoio a programas de cooperação técnica internacional entre o Reino Unido e o Brasil, coordenados pelo Ministério da Saúde. Ainda assim existe a necessidade de maior consolidação dessa área no Brasil.

Este artigo identifica a capacidade de produção de conhecimento, bem como os estudos já produzidos relacionados à ES no Brasil. Seus resultados permitem vislumbrar a riqueza, a magnitude e as potencialidades observadas no processo de construção desse campo de conhecimento. O 
conteúdo apresentado aqui é parte integrante de uma análise situacional que enfoca mais amplamente o desenvolvimento da área em nosso país (Andrade et al., 2004).

\section{Metodologia}

A capacidade de produção científica na área de economia da saúde, no Brasil, foi analisada mapeando-se os grupos de pesquisa cadastrados no Conselho Nacional de Desenvolvimento Científico e Tecnológico (CNPq) que desenvolvem trabalhos pertinentes à área. Também foi realizado um mapeamento bibliográfico de produções acadêmicas, científicas e técnicas relacionadas à avaliação tecnológica em saúde (ATS) e ES, por meio de busca em bancos de dados informatizados.

A produção científica identificada foi classificada segundo áreas temáticas. No processo de definição dessas áreas, procurou-se associar questões relacionadas ao papel dos serviços de saúde no sistema econômico, enfocando estudos que compreendam não só análise de custos, custo-benefício e custo-efetividade, como também análises relativas à acessibilidade econômica a bens e serviços de saúde, à eficiência e efetividade na gestão das políticas públicas de saúde, ao mercado farmacêutico e ao mercado de saúde suplementar, desde que pudessem se constituir em subsídios para o processo de tomada de decisão (Lucchese, 2003). Assim, o presente estudo adotou as seguintes áreas temáticas:

․ medicamentos, insumos e assistência farmacêutica;

equipamentos médicos e hospitalares;

a equipamentos e métodos de diagnóstico;

ㄱ procedimentos clínicos e cirúrgicos;

[ educação sanitária e promoção da saúde;

u financiamento, alocação e eqüidade;

․ inovação tecnológica.

A composição de cada área temática é apresentada no quadro 1. 


\begin{tabular}{|c|c|}
\hline \multicolumn{2}{|c|}{$\begin{array}{c}\text { Quadro } 1 \\
\text { Áreas temáticas utilizadas para a classificação da produção } \\
\text { científica em economia da saúde }\end{array}$} \\
\hline Áreas temáticas & Composição \\
\hline $\begin{array}{l}\text { Medicamentos, insumos } \\
\text { e assistência farmacêutica }\end{array}$ & $\begin{array}{l}\text { Estudos contendo avaliações econômicas aplicadas aos medicamentos, insumos } \\
\text { farmacêuticos e substâncias com finalidade terapêutica e diagnóstica, bem como } \\
\text { estudos que enfoquem repercussões econômicas realizados no campo da } \\
\text { assistência farmacêutica. }\end{array}$ \\
\hline $\begin{array}{l}\text { Equipamentos médicos } \\
\text { e hospitalares }\end{array}$ & $\begin{array}{l}\text { Estudos contendo avaliações tecnológicas e econômicas sobre recursos indiretos } \\
\text { para realização dos procedimentos médicos, compreendendo as órteses, próteses } \\
\text { e equipamentos de suporte terapêutico, excluídos os equipamentos para } \\
\text { diagnóstico. }\end{array}$ \\
\hline $\begin{array}{l}\text { Equipamentos e métodos } \\
\text { de diagnóstico }\end{array}$ & $\begin{array}{l}\text { Estudos contendo avaliações tecnológicas e econômicas sobre os métodos e } \\
\text { equipamentos utilizados com finalidade diagnóstica. }\end{array}$ \\
\hline $\begin{array}{l}\text { Procedimentos clínicos } \\
\text { e cirúrgicos }\end{array}$ & $\begin{array}{l}\text { Estudos contendo avaliações econômicas sobre gastos e custos de } \\
\text { procedimentos clínicos e cirúrgicos, incluindo estudos relativos à padronização de } \\
\text { protocolos e guias terapêuticos, bem como estudos que discutam ou apresentem } \\
\text { repercussões econômicas realizados no campo da regulação do setor saúde, } \\
\text { especificamente, sobre procedimentos clínicos e cirúrgicos. }\end{array}$ \\
\hline $\begin{array}{l}\text { Educação sanitária e } \\
\text { promoção da saúde }\end{array}$ & $\begin{array}{l}\text { Estudos contendo avaliações econômicas sobre políticas, projetos e ações } \\
\text { realizadas com finalidade de promover a saúde da população por meio de ações } \\
\text { preventivas e da educação sanitária. }\end{array}$ \\
\hline $\begin{array}{l}\text { Gestão, financiamento, } \\
\text { alocação e eqüidade }\end{array}$ & $\begin{array}{l}\text { Estudos contendo avaliações econômicas sobre modelos de gestão de sistemas e } \\
\text { serviços, de políticas públicas em saúde, bem como avaliações sobre } \\
\text { financiamento, investimento, orçamento, gasto e eqüidade na distribuição dos } \\
\text { recursos destinados à saúde. }\end{array}$ \\
\hline Inovação tecnológica & $\begin{array}{l}\text { Estudos contendo avaliações sobre o investimento em P\&D em saúde e no setor } \\
\text { biomédico, correlacionados com a formação de recursos humanos, produção } \\
\text { científica e a propriedade intelectual/industrial. }\end{array}$ \\
\hline
\end{tabular}

\section{Mapeamento de grupos de pesquisa}

Investigaram-se informações sobre recursos humanos constituintes desses grupos, linhas de pesquisa em andamento, especialidades do conhecimento, setores de aplicação envolvidos, produção científica e tecnológica, padrões 
de interação com o setor produtivo, bem como o tipo de repercussão obtida com as atividades realizadas.

Para tanto, foi realizada uma busca textual na base de dados do Diretório de Grupos de Pesquisa da Plataforma Lattes certificados no Brasil, mantido pelo Conselho Nacional de Desenvolvimento Científico e Tecnológico (CNPq), do Ministério da Ciência e Tecnologia (MCT) e disponibilizado na internet (CNPq, 2004). Os grupos constantes nessa base de dados podem estar localizados em universidades, instituições isoladas de ensino superior, institutos de pesquisa científica, institutos tecnológicos, laboratórios de pesquisa e desenvolvimento de empresas estatais ou ex-estatais e em organizações não-governamentais com atuação em pesquisa científica ou tecnológica. Os dados disponibilizados não incluem grupos de pesquisa localizados em empresas do setor produtivo privado.

Para o processo de busca dos grupos de pesquisa, foram estabelecidos os seguintes critérios:

(] palavras-chave - busca de grupos associados na base do CNPq às palavras-chave elencadas no quadro 2 . Essas palavras-chave foram estabelecidas após busca no sistema de terminologia em saúde, "Descritores em Ciência da Saúde" (Desc), e complementadas com outros termos utilizados na área. Esse sistema utiliza vocabulários estruturados, que são coleções de termos, organizados segundo uma metodologia na qual é possível especificar as relações entre conceitos, com o propósito de facilitar o acesso à informação;

a seleção de grupos de interesse - foram relacionados os grupos de pesquisa obtidos no processo de busca, e escolhidos apenas aqueles cuja informação registrada pelo líder do grupo no campo "repercussões dos trabalhos do grupo" estava relacionada às áreas temáticas de interesse definidas no quadro 1 para este estudo;

a validação das informações sobre grupos - com o intuito de confirmar as informações obtidas, os grupos selecionados foram contatados a partir dos líderes ou pessoa por ele indicada, via telefone. Complementarmente procurou-se atualizar informações sobre os participantes do grupo, os estudos em andamento e os já concluídos;

a levantamento do perfil dos colaboradores - com as informações sobre os participantes do grupo que efetivamente trabalham em economia da saúde validadas pelos líderes, buscou-se identificar o perfil de formação das pessoas que estão atuando na área, por meio do curriculum Lattes. 
O período de coleta de dados referentes aos grupos de pesquisa na Plataforma Lattes compreendeu os meses de março e abril de 2004, e a validação das informações realizada, via contato telefônico, de maio a julho de 2004 .

\begin{tabular}{|c|c|}
\hline \multicolumn{2}{|c|}{$\begin{array}{c}\text { Quadro } 2 \\
\text { Palavras-chave utilizadas para pesquisa em bases de dados }\end{array}$} \\
\hline Palavras-chave & Termos para busca* \\
\hline Acessibilidade econômica & Acess $\$$ econom $\$$ \\
\hline Alocação & Alocaç\$ \\
\hline Análise de custos & Análise $\$$ custo $\$$ \\
\hline Análise de impacto no orçamento & Análise \$ de impacto no orcament\$ \\
\hline Análise de sensibilidade & Análise $\$$ de sensibilidade\$ \\
\hline Análise econômica & Análise\$ economic\$ \\
\hline Custo da doença & Custo\$ da doença\$ \\
\hline Custo-benefício & Custo $\$$ benefício $\$$ \\
\hline Custo-efetividade & Custo $\$$ efetividade $\$$ \\
\hline Custo-eficiência & Custo\$ eficiência\$ \\
\hline Custo-oportunidade & Custo\$ oportunidade\$ \\
\hline Custo-utilidade & Custo\$ utilidade\$ \\
\hline Economia da saúde & Econom $\$$ da saúde\$ \\
\hline Farmacoeconomia & Farmacoeconom\$ \\
\hline Financiamento & Financia\$ \\
\hline Gastos & Gasto $\$$ \\
\hline Avaliação econômica & Avalia $\$$ econom\$ \\
\hline
\end{tabular}

\section{Produção científica e técnica}

Foram catalogados resumos de artigos, dissertações, teses, monografias, livros e outros documentos oficiais que enfocaram a área de ES e avaliação tecnológica em saúde no Brasil. Para mapear essa produção científica, foi realizada uma busca ativa nas bases de dados informatizadas disponíveis na Biblioteca Virtual em Saúde mantidas pela Bireme (2004), a saber:

․ Lilacs - literatura latino-americana e do Caribe em ciências da saúde;

૫ Medline - literatura internacional em ciências da saúde e biomédica; 
[ Adolec — saúde na adolescência;

$\quad \mathrm{BBO}$ - bibliografia brasileira de odontologia;

․ Bdenf — base de dados de enfermagem;

Hisa - história da saúde pública na América Latina e Caribe;

․ Leyes - legislação básica de saúde da América Latina e Caribe;

a Medcarib - literatura do Caribe em ciências da saúde;

․ Repidisca - literatura em engenharia sanitária e ciências do ambiente;

․ Desastres - acervo do Centro de Documentação de Desastres;

[ Opas - acervo da biblioteca da Organização Pan-Americana da Saúde;

口 Wholis — sistema de informação da biblioteca da Organização Mundial de Saúde.

Para o processo de busca textual da produção científica, foram estabelecidos os seguintes critérios:

a palavras-chave - busca ativa de informações a partir dos termos de busca relacionados às palavras-chave no quadro 2 ;

口 seleção de documentos de interesse - os documentos obtidos no processo de busca ativa associados às palavras-chave alimentaram uma base de dados, organizada, preliminarmente, em planilha do programa Microsoft Excel ${ }^{\circledR} 2000$;

u classificação - os trabalhos foram avaliados de acordo com o campo da economia da saúde ao qual se relacionam, a partir das informações disponibilizadas nos respectivos resumos. Classificaram-se os documentos conforme a área temática, o tipo de estudo e a abrangência dos dados (multicêntrico, nacional, estadual, municipal, local e unidade).

O período de coleta de dados na página da BVS/Bireme, por meio de busca ativa na internet, ocorreu entre maio e julho de 2004. Foram catalogados documentos publicados entre janeiro de 1999 e julho de 2004. O processo de análise, classificação e constituição do banco de dados foi realizado nos meses de junho e julho de 2004. 


\section{Tratamento e análise dos dados}

Os dados obtidos foram descritos e organizados em tabelas de distribuições de freqüências e em gráficos. Foram estabelecidos os seguintes indicadores que, em conjunto, contribuem para estabelecer uma análise sobre a situação da área de economia da saúde no país:

ㄱ número de grupos de pesquisa com repercussões em ES e ATS;

percentual desses grupos de pesquisa em relação ao total de grupos do CNPq;

口 número de grupos de pesquisa por estado;

口 percentual de grupos de pesquisa por estado em relação ao total de grupos (ES e ATS);

․ número de grupos de pesquisa por região;

口 percentual de grupos de pesquisa por região em relação ao total de grupos (ES e ATS);

૫ perfil da formação acadêmica de pesquisadores desses grupos de pesquisa;

․ perfil das linhas de pesquisa desses grupos;

u produção do conhecimento em ES e ATS;

[ ] produção por área temática em relação à produção total mapeada;

[ ] produção por abrangência dos dados em relação à produção total mapeada;

[ produção por tipo de estudo em relação à produção total mapeada;

[ produção por instituição em relação à produção total mapeada;

[ produção por região do país em relação à produção total mapeada;

[ produção por veículo de publicação em relação à produção total mapeada.

\section{Resultados}

\section{Grupos de pesquisa}

Inventariaram-se 175 grupos diferentes de pesquisa relacionados às palavras no quadro 2, cadastrados no CNPq/MCT. Esses grupos foram submetidos a um processo de seleção por meio de análise do campo "repercussões do grupo" na base de dados, e classificados segundo seu perfil e correlação com a área de econo- 
mia da saúde. Após o processo de validação, obteve-se um total de 48 grupos cujos trabalhos estão relacionados às áreas temáticas referentes à ES. Na base de dados da Plataforma Lattes do CNPq/MCT estão registrados 15.158 grupos de pesquisa, sendo que $4.914(32,4 \%)$ desse total atuam na área de saúde. Os grupos com repercussões na área de ES representam apenas 0,3\% do total de grupos dessa base e $1 \%$ dos grupos atuantes na área de saúde.

Os 48 grupos de pesquisa avaliados têm escopo de atuação diversificado. A maioria (96\%) desenvolve atividades de pesquisa, 94\% realizam orientações em trabalhos de pós-graduação (monografias, dissertações e teses), 77\% exercem atividades de consultoria e $58 \%$ oferecem cursos de extensão.

Quanto aos tipos de estudos desenvolvidos pelos grupos, 69\% realizam estudos de análise de custos; 63\%, estudos sobre eficácia, efetividade e eficiência na gestão; 58\%, estudos sobre acessibilidade econômica e análises de custo - efetividade; e $56 \%$, estudos sobre análise de custo-benefício. Além disso, 39\% dos grupos desenvolvem análises de custo da doença; $37 \%$ realizam estudos sobre mercado farmacêutico e análise de sustentabilidade; 35\% de análises de minimização de custos; 33\% de análise de custo-utilidade; e $31 \%$ de estudos sobre o mercado de saúde suplementar.

A classificação de acordo com as áreas temáticas de atuação dos grupos de pesquisa é apresentada na tabela 1, onde observa-se que 73\% indicaram atuação na área de gestão em saúde; $63 \%$ na área farmacêutica; $58 \%$ na de financiamento, alocação e eqüidade; $52 \%$ na de educação sanitária e promoção da saúde; e 48\% em inovação tecnológica.

A distribuição dos grupos de pesquisa (ES e ATS), segundo a instituição de origem e unidade da Federação, mostra que 14 grupos (30\%) encontram-se no estado de São Paulo, sendo metade mantida pela Universidade de São Paulo. Os demais $(n=7)$ estão distribuídos em diversas instituições do estado: Instituto Adolfo Lutz, Instituto de Saúde da Secretaria do Estado de Saúde de São Paulo, Pontifícia Universidade Católica de Campinas, Universidade de Campinas, Universidade Federal de São Paulo, Universidade Metodista de Piracicaba, Universidade de Sorocaba e Universidade Comunitária Regional de Chapecó.

No estado do Rio de Janeiro estão localizados 11 (23\%) dos grupos de pesquisa avaliados, sendo oito mantidos pela Fundação Oswaldo Cruz e os demais distribuídos na Universidade Estadual do Rio de Janeiro, Universidade Federal Fluminense e Universidade Federal do Rio de Janeiro.

Em terceiro lugar, Minas Gerais e Rio Grande do Sul mantêm igual número de grupos de pesquisa $(n=4)$, cada unidade apresentando $9 \%$ do conjunto nacional. A Universidade Federal de Minas Gerais mantém três grupos e a Universidade Federal de Juiz de Fora, um grupo. A Universidade Federal do Rio Grande do Sul mantém três grupos e a Universidade Católica de Pelotas, um grupo. 
Os demais grupos, representando 30\% do total, estão distribuídos pelos seguintes estados e instituições: no Paraná, Pontifícia Universidade do Paraná e Universidade Estadual de Londrina; no Ceará, Universidade Federal do Ceará e Universidade Estadual do Ceará; na Paraíba, Universidade Federal da Paraíba e Universidade Estadual da Paraíba; na Bahia, Universidade Federal da Bahia e Universidade Estadual de Feira de Santana; em Santa Catarina, Universidade Comunitária Regional de Chapecó; no Rio Grande do Norte, Universidade Federal do Rio Grande do Norte; em Pernambuco, Instituto Materno Infantil de Pernambuco; e no Mato Grosso do Sul, Universidade Federal do Mato Grosso do Sul.

Observa-se uma concentração de grupos atuantes em economia da saúde na região Sudeste, onde estão localizados 64\% dos grupos de pesquisa. Em seguida, vem a região Nordeste, com 19\% dos grupos, seguida pela região Sul com $15 \%$ e pela região Centro-Oeste, com apenas $2 \%$ dos grupos selecionados.

A partir do diretório de grupos de pesquisa da Plataforma Lattes - $\mathrm{CNPq} /$ MCT foram identificadas e validadas 82 linhas de pesquisa (detalhamento não apresentado) desenvolvidas pelos grupos com repercussão em ES.

\begin{tabular}{|c|c|c|}
\hline \multicolumn{3}{|c|}{$\begin{array}{c}\text { Tabela } 3 \\
\text { Áreas temáticas de atuação dos grupos de pesquisa }(n=48)\end{array}$} \\
\hline Áreas temáticas & $n$ & $\%$ \\
\hline Gestão em saúde & 35 & 73 \\
\hline Medicamentos, insumos, assistência farmacêutica & 30 & 63 \\
\hline Financiamento, alocação, eqüidade & 28 & 58 \\
\hline Educação sanitária e promoção da saúde & 25 & 52 \\
\hline Inovação tecnológica & 23 & 48 \\
\hline Equipamentos e métodos de diagnóstico & 20 & 42 \\
\hline Procedimentos clínicos e cirúrgicos & 19 & 40 \\
\hline Equipamentos médicos e hospitalares & 13 & 27 \\
\hline Outras & 11 & 23 \\
\hline
\end{tabular}

\section{Perfil dos pesquisadores}

Foram identificados 324 pesquisadores pertencentes aos grupos de pesquisa selecionados, sendo que 14 deles se inseriram em mais de um grupo e 38 não 
tinham o registro de suas informações acadêmicas na base de currículos Lattes. Portanto, a análise do perfil acadêmico incluiu informações relativas a 272 pesquisadores atuantes em economia da saúde.

Entre as áreas de conhecimento identificadas, observa-se uma predominância de pesquisadores advindos da área de ciências da saúde, perfazendo $67 \%$ do total de pesquisadores analisados, como demonstrado na figura 1 . Ressalta-se a discrepância existente entre a atuação de profissionais da área de ciências da saúde e das demais áreas de conhecimento. Segue-se a composição da área de ciências da saúde, que está representada pelos seguintes cursos: medicina ( $45 \%$ dos pesquisadores), farmácia (29\%), odontologia (14\%), enfermagem ( $8 \%)$, fisioterapia ( $2 \%)$ e nutrição $(2 \%)$.

A área de medicina prevalece e a de ciências sociais aplicadas é a segunda área de conhecimento com maior participação de profissionais de ES (10\%). Nessa área, observa-se evidente maioria de profissionais de ciências econômicas (59\%), seguidos pelos graduados em administração (28\%), direito (10\%) e arquitetura e urbanismo (3\%).

Quanto à distribuição da titulação máxima obtida pelos 272 pesquisadores, observa-se que $34(12,5 \%)$ possuem pós-doutorado, 141 (51,8\%), doutorado, $72(26,5 \%)$, mestrado, enquanto $18(6,6 \%)$ possuem especialização e sete $(2,6 \%)$ não realizaram nenhum curso de pós-graduação.

\section{Figura 1}

Distribuição de pesquisadores por área de conhecimento $(n=272)$

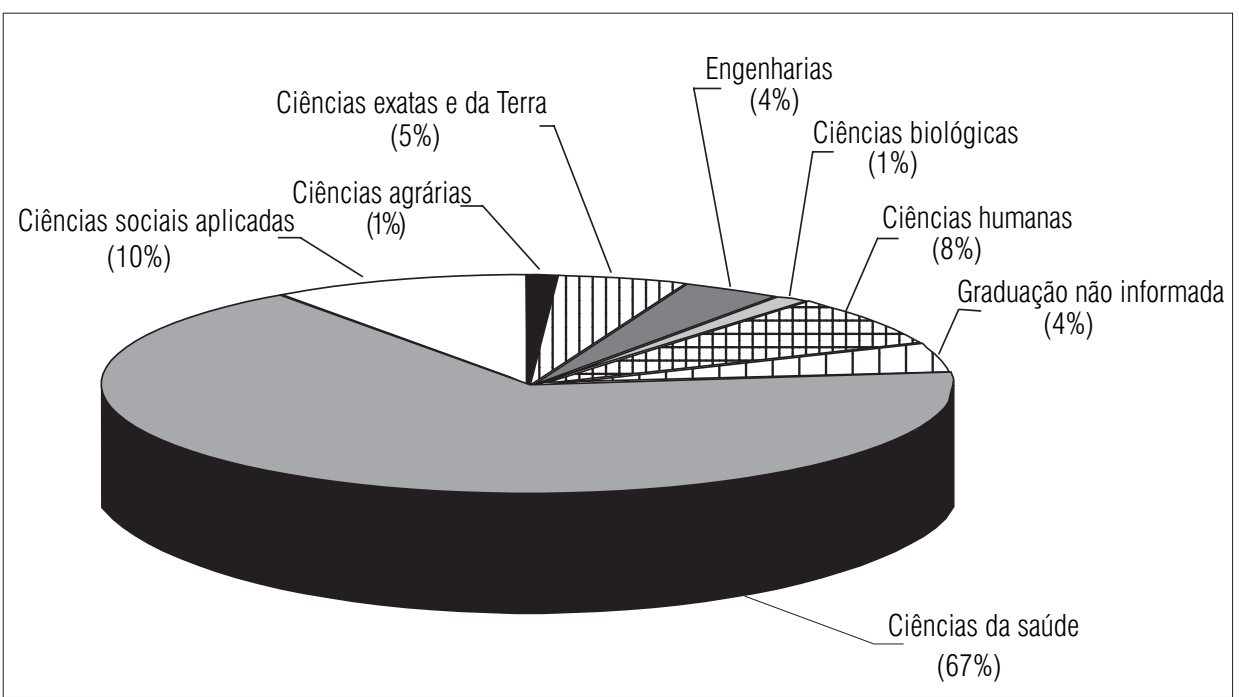




\section{Produção científica e técnica}

Foram capturados preliminarmente 5.239 trabalhos, utilizando-se o critério de busca proposto para as publicações na BVS/Bireme. Especificamente para a base de dados Medline, acrescentou-se um filtro ao processo de pesquisa, inventariando-se somente os trabalhos que continham a palavra Brasil ou Brazil nos campos descritores. A leitura, avaliação e posterior classificação das publicações foram feitas apenas para aqueles documentos que continham resumo, o que representou a seleção de 2.617 documentos. Destes, $376(14,3 \%)$ estavam relacionados à área de economia da saúde e foram considerados para a construção do perfil de publicações.

As áreas temáticas às quais se relacionam as publicações em ES estão apresentadas na tabela 2. Observa-se a predominância de estudos que enfocam gestão, financiamento, alocação e eqüidade na distribuição de recursos, representando $48 \%$ do total de documentos.

Os documentos foram classificados pelo tipo de estudo, observando-se que, do total, $34,6 \%$ das publicações se referem a gestão em saúde e/ou políticas públicas, sendo esse o enfoque dos livros e teses. O foco da maioria dos artigos se distribui de forma proporcional entre estudos sobre gestão em saúde e/ou políticas públicas, com 70 publicações; e análise de custos e gastos em saúde, com 68 documentos. Verifica-se que $38 \%$ das publicações são referentes a estudos que utilizam ferramentas de avaliação econômica, sendo que o enfoque foi dado à análise de custos e gastos em saúde.

A distribuição das publicações por origem revela que as instituições que apresentam maior número de publicações são a Universidade de São Paulo $(n=71)$, a Fundação Oswaldo Cruz $(n=47)$, a Universidade do Estado do Rio de Janeiro $(n=21)$, a Universidade Federal de São Paulo $(n=21)$, a Fundação Getulio Vargas $(n=18)$, o Instituto de Pesquisa Econômica Aplicada $(n=11)$, o Ministério da Saúde $(n=10)$, a Universidade Federal do Rio de Janeiro $(n=10)$, a Pontifícia Universidade Católica de São Paulo $(n=7)$, a Universidade Federal de Minas Gerais $(n=6)$ e a Universidade Estadual Paulista $(n=6)$. Em conjunto, essas instituições são responsáveis por $60,6 \%$ das 376 publicações relacionadas. Observa-se, na tabela 2, a predominância de documentos acadêmicos (universidade, centros universitários, escolas e faculdades) com $60,1 \%$ das publicações, seguidos pelos institutos de pesquisa, com $21,3 \%$ dos documentos.

Por meio das instituições de origem, identificaram-se os estados de proveniência das publicações, com maior freqüência de publicações $(77,0 \%)$ verificada na região Sudeste.

Os 10 principais veículos de publicação utilizados para a divulgação da produção científica (artigos, livros e teses) são apresentados na tabela 3. 
Na distribuição das publicações segundo o ano calendário (tabela 2), observa-se um acréscimo de $102 \%$ da produção de 1999 para a de 2002, com um decréscimo de $30 \%$ deste ano para 2003. As publicações se concentraram no ano de 2002, quando produziu-se $26,3 \%$ do total de publicações analisadas.

Na tabela 2 apresenta-se a produção científica segundo a abrangência dos dados, observando-se que $30 \%$ das informações utilizadas pelos pesquisadores nos respectivos estudos eram de abrangência nacional. Os dados referentes às unidades de serviço mostram a segunda abrangência com maior representatividade $(17,3 \%)$. Constata-se que houve estudos nas diversas abrangências definidas, embora os estudos locais representem $0,8 \%$ do total.

\begin{tabular}{|c|c|c|c|c|c|c|c|c|}
\hline \multicolumn{9}{|c|}{$\begin{array}{c}\text { Tabela } 2 \\
\text { Produção científica (artigos, livros e teses), segundo } \\
\text { características selecionadas }\end{array}$} \\
\hline \multirow[b]{2}{*}{ Características } & \multicolumn{2}{|c|}{ Artigo } & \multicolumn{2}{|c|}{ Livro } & \multicolumn{2}{|c|}{ Tese } & \multicolumn{2}{|c|}{ Total } \\
\hline & $n$ & $\%$ & $n$ & $\%$ & $n$ & $\%$ & $n$ & $\%$ \\
\hline Área temática & 241 & 100,0 & 43 & 100,0 & 92 & 100,0 & 376 & 100,0 \\
\hline $\begin{array}{l}\text { Gestão, financiamento, alocação, } \\
\text { eqüidade }\end{array}$ & 107 & 44,4 & 27 & 62,8 & 46 & 50,0 & 180 & 47,9 \\
\hline $\begin{array}{l}\text { Medicamentos, insumos, assistência } \\
\text { farmacêutica }\end{array}$ & 41 & 17,0 & 1 & 2,3 & 15 & 16,3 & 57 & 15,2 \\
\hline P Procedimentos clínicos e cirúrgicos & 46 & 19,1 & 0 & 0,0 & 9 & 9,8 & 55 & 14,6 \\
\hline ૨ Educação sanitária e promoção da saúde & 13 & 5,4 & 8 & 18,6 & 7 & 7,6 & 28 & 7,4 \\
\hline ૫ Equipamentos e métodos de diagnóstico & 11 & 4,6 & 0 & 0,0 & 2 & 2,2 & 13 & 3,5 \\
\hline ૫ Inovação tecnológica & 7 & 2,9 & 0 & 0,0 & 1 & 1,1 & 8 & 2,1 \\
\hline Equipamentos médicos e hospitalares & 1 & 0,4 & 0 & 0,0 & 1 & 1,1 & 2 & 0,5 \\
\hline Outras & 15 & 6,2 & 7 & 16,3 & 11 & 12,0 & 33 & 8,8 \\
\hline Tipo de estudo & 241 & 100,0 & 43 & 100,0 & 92 & 100,0 & 376 & 100,0 \\
\hline $\begin{array}{l}\text { Estudos sobre gestão em saúde e/ou } \\
\text { políticas públicas }\end{array}$ & 70 & 29,0 & 25 & 58,1 & 35 & 38,0 & 130 & 34,6 \\
\hline ૫ Análise de custos e gastos em saúde & 68 & 28,2 & 5 & 11,6 & 15 & 16,3 & 88 & 23,4 \\
\hline ૫ Análise de custo-efetividade & 20 & 8,3 & 1 & 2,3 & 2 & 2,2 & 23 & 6,1 \\
\hline Estudos sobre mercado de saúde & 7 & 2,9 & 6 & 14,0 & 10 & 10,9 & 23 & 6,1 \\
\hline $\begin{array}{l}\text { Estudos sobre acesso e/ou } \\
\text { acessibilidade econômica }\end{array}$ & 17 & 7,1 & 0 & 0,0 & 5 & 5,4 & 22 & 5,9 \\
\hline
\end{tabular}




\begin{tabular}{|c|c|c|c|c|c|c|c|c|}
\hline \multirow[b]{2}{*}{ Características } & \multicolumn{2}{|c|}{ Artigo } & \multicolumn{2}{|c|}{ Livro } & \multicolumn{2}{|c|}{ Tese } & \multicolumn{2}{|c|}{ Total } \\
\hline & $n$ & $\%$ & $n$ & $\%$ & $n$ & $\%$ & $n$ & $\%$ \\
\hline ૫ Análise de custo da doença & 13 & 5,4 & 0 & 0,0 & 3 & 3,3 & 16 & 4,3 \\
\hline 口 Análise de custo-benefício & 13 & 5,4 & 0 & 0,0 & 2 & 2,2 & 15 & 4,0 \\
\hline c Análise de impacto no orçamento & 5 & 2,1 & 2 & 4,7 & 0 & 0,0 & 7 & 1,9 \\
\hline ૫ Estudos sobre mercado farmacêutico & 3 & 1,2 & 0 & 0,0 & 3 & 3,3 & 6 & 1,6 \\
\hline ૫ Análise de custo-oportunidade & 0 & 0,0 & 0 & 0,0 & 1 & 1,1 & 1 & 0,3 \\
\hline ૫ Análise de custo-utilidade & 1 & 0,4 & 0 & 0,0 & 0 & 0,0 & 1 & 0,3 \\
\hline Outros & 24 & 10,0 & 4 & 9,3 & 16 & 17,4 & 44 & 11,7 \\
\hline Tipo de instituição & 241 & 100,0 & 43 & 100,0 & 92 & 100,0 & 376 & 100,0 \\
\hline ૫ Academia & 154 & 63,9 & 10 & 23,3 & 62 & 67,4 & 226 & 60,1 \\
\hline I Instituto de pesquisa & 35 & 14,5 & 15 & 34,9 & 30 & 32,6 & 80 & 21,3 \\
\hline ૧ Administração pública & 16 & 6,6 & 8 & 18,6 & 0 & 0,0 & 24 & 6,4 \\
\hline ૫ Organismo internacional & 11 & 4,6 & 0 & 0,0 & 0 & 0,0 & 11 & 2,9 \\
\hline Setor privado & 2 & 0,8 & 0 & 0,0 & 0 & 0,0 & 2 & 0,5 \\
\hline Outros & 17 & 7,1 & 3 & 7,0 & 0 & 0,0 & 20 & 5,3 \\
\hline ૫ Não localizado & 6 & 2,5 & 7 & 16,3 & 0 & 0,0 & 13 & 3,5 \\
\hline Região* & 191 & 100,0 & 43 & 100,0 & 92 & 100,0 & 326 & 100,0 \\
\hline C Centro-Oeste & 10 & 5,2 & 11 & 25,6 & 1 & 1,1 & 22 & 6,7 \\
\hline Nordeste & 18 & 9,4 & 3 & 7,0 & 3 & 3,3 & 24 & 7,4 \\
\hline N Norte & 1 & 0,5 & 1 & 2,3 & 0 & 0,0 & 2 & 0,6 \\
\hline ( Sudeste & 144 & 75,4 & 20 & 46,5 & 87 & 94,6 & 251 & 77,0 \\
\hline 口 Sul & 18 & 9,4 & 1 & 2,3 & 1 & 1,1 & 20 & 6,1 \\
\hline — Não localizado & 0 & 0,0 & 7 & 16,3 & 0,0 & 0,0 & 7 & 2,1 \\
\hline Ano de publicação & 241 & 100,0 & 43 & 100,0 & 92 & 100,0 & 376 & 100,0 \\
\hline 口 1999 & 27 & 11,2 & 10 & 23,3 & 12 & 13,0 & 49 & 13,0 \\
\hline ㄱ 2000 & 47 & 19,5 & 10 & 23,3 & 9 & 9,8 & 66 & 17,6 \\
\hline ㄴ 2001 & 41 & 17,0 & 17 & 39,5 & 24 & 26,1 & 82 & 21,8 \\
\hline ㄴ 2002 & 59 & 24,5 & 5 & 11,6 & 35 & 38,0 & 99 & 26,3 \\
\hline ㄴ 2003 & 56 & 23,2 & 1 & 2,3 & 12 & 13,0 & 69 & 18,4 \\
\hline ㄷ $2004^{* *}$ & 11 & 4,6 & 0 & 0,0 & 0 & 0,0 & 11 & 2,9 \\
\hline Abrangência dos dados & 241 & 100,0 & 43 & 100,0 & 92 & 100,0 & 376 & 100,0 \\
\hline ૫ Multicêntrica & 38 & 15,8 & 1 & 2,3 & 2 & 2,2 & 41 & 10,9 \\
\hline ( Nacional & 70 & 29,0 & 15 & 34,9 & 31 & 33,7 & 116 & 30,9 \\
\hline ૫ Estadual & 16 & 6,6 & 4 & 9,3 & 6 & 6,5 & 26 & 6,9 \\
\hline
\end{tabular}




\begin{tabular}{|c|c|c|c|c|c|c|c|c|}
\hline \multirow[b]{2}{*}{ Características } & \multicolumn{2}{|c|}{ Artigo } & \multicolumn{2}{|c|}{ Livro } & \multicolumn{2}{|c|}{ Tese } & \multicolumn{2}{|c|}{ Total } \\
\hline & $n$ & $\%$ & $n$ & $\%$ & $n$ & $\%$ & $n$ & $\%$ \\
\hline ( Municipal & 34 & 14,1 & 2 & 4,7 & 26 & 28,3 & 62 & 16,5 \\
\hline c Local & 3 & 1,2 & 0 & 0,0 & 0 & 0,0 & 3 & 0,8 \\
\hline ૫ Unidade & 45 & 18,7 & 2 & 4,7 & 18 & 19,6 & 65 & 17,3 \\
\hline 口 Outra & 35 & 14,5 & 19 & 44,2 & 9 & 9,8 & 63 & 16,8 \\
\hline
\end{tabular}

\begin{tabular}{|c|c|c|c|c|}
\hline \multicolumn{5}{|c|}{$\begin{array}{c}\text { Tabela } 3 \\
\text { Veículos de publicação com maior produção científica } \\
\text { (livros, teses e artigos) }\end{array}$} \\
\hline Tipo de publicação & Veículos de publicação & $n$ & $\%$ & $\% A C$ \\
\hline \multirow[t]{12}{*}{ Artigo } & Total de artigos & 241 & 100,0 & - \\
\hline & Cad. Saúde Pública & 32 & 13,3 & 13,3 \\
\hline & Ciênc. Saúde Coletiva & 22 & 9,1 & 22,4 \\
\hline & Rev. Panam. Salud Publica & 15 & 6,2 & 28,6 \\
\hline & Rev. Assoc. Med. Bras. & 10 & 4,1 & 32,8 \\
\hline & Arq. Bras. Oftalmol. & 8 & 3,3 & 36,1 \\
\hline & RAP & 6 & 2,5 & 38,6 \\
\hline & Saúde Soc. & 6 & 2,5 & 41,1 \\
\hline & Bioética & 5 & 2,1 & 43,2 \\
\hline & Mundo Saúde (1995) & 5 & 2,1 & 45,2 \\
\hline & Rev. Saúde Pública & 5 & 2,1 & 47,3 \\
\hline & Restante dos artigos em conjunto & 127 & 52,7 & 100,0 \\
\hline \multirow[t]{10}{*}{ Livros } & Total de livros & 43 & 100,0 & - \\
\hline & Ipea. Texto para discussão & 9 & 20,9 & 20,9 \\
\hline & Editora Atlas & 4 & 9,3 & 30,2 \\
\hline & Ministério da Saúde & 3 & 7,0 & 37,2 \\
\hline & Fundação Getulio Vargas — Escola de & & & \\
\hline & Administração de Empresas de São Paulo & 2 & 4,7 & 41,9 \\
\hline & Gazeta Mercantil Informações Eletrônicas — & & & \\
\hline & Análise setorial & 2 & 4,7 & 46,5 \\
\hline & Ministério da Saúde — Coordenação & & & \\
\hline & Nacional de DST e Aids & 2 & 4,7 & 51,2 \\
\hline
\end{tabular}




\begin{tabular}{|c|c|c|c|c|}
\hline Tipo de publicação & Veículos de publicação & $n$ & $\%$ & $\% A C$ \\
\hline & Universidade do Estado do Rio de Janeiro - & 2 & 47 & 558 \\
\hline & ANS. Série C. Projetos, Programas e Relatórios & 1 & 2,3 & 58,1 \\
\hline & Casa da Qualidade Editora & 1 & 2,3 & 60,5 \\
\hline & Centro Universitário São Camilo — Coleção & & & \\
\hline & Bioética em Perspectiva & 1 & 2,3 & 62,8 \\
\hline & Restante dos livros em conjunto & 16 & 37,2 & 100,0 \\
\hline \multirow[t]{19}{*}{ Teses } & Total de teses & 92 & 100,0 & - \\
\hline & Escola de Administração de Empresas & & & \\
\hline & de São Paulo & 14 & 15,2 & 15,2 \\
\hline & Escola Nacional de Saúde Pública & 14 & 15,2 & 30,4 \\
\hline & $\begin{array}{l}\text { Universidade de São Paulo — Faculdade } \\
\text { de Saúde Pública }\end{array}$ & 14 & 15.2 & 45.7 \\
\hline & Universidade do Estado do Rio de Janeiro - & & & \\
\hline & Instituto de Medicina Social & 14 & 15,2 & 60,9 \\
\hline & Universidade de São Paulo — Escola de & & & \\
\hline & Enfermagem & 4 & 4,3 & 65,2 \\
\hline & Universidade de São Paulo — Faculdade & & & \\
\hline & de Saúde Pública & 3 & 3,3 & 68,5 \\
\hline & Centro de Pesquisas Aggeu Magalhães & 2 & 2,2 & 70,7 \\
\hline & Universidade de São Paulo — Faculdade de & & & \\
\hline & Ciências Farmacêuticas & 2 & 2,2 & 72,8 \\
\hline & Universidade de São Paulo — Faculdade & & & \\
\hline & de Odontologia & 2 & 2,2 & 75,0 \\
\hline & Universidade de São Paulo — Faculdade & & & \\
\hline & de Saúde Pública & 2 & 2,2 & 77,2 \\
\hline & Restante das teses em conjunto & 21 & 22,8 & 100,0 \\
\hline
\end{tabular}

Em 1998, a evolução da produção científica em economia da saúde no Brasil foi sistematizada por Vianna, que encontrou 185 títulos publicados entre 1986 e 1995. Em estudo mais recente, Saes (2000) analisou o estadoda-arte desse campo de conhecimento no país, pesquisando publicações no período de 1989 a 1998. O escopo dos descritores para a área de ES utilizado por tal autora tem semelhança com o presente artigo, o que agrega maior homogeneidade à análise. Pode-se observar na figura 2 um crescimento contínuo da produção científica em ES no Brasil, até 2002. A despeito da queda em 2003, a produção naquele ano encontra-se em patamar mais elevado do que o observado no período de 1999 a 2000. 
Figura 2

Evolução da produção científica em economia da saúde no Brasil por ano baseada nos estudos de Saes (2000) e GPES - UFMG (2004)

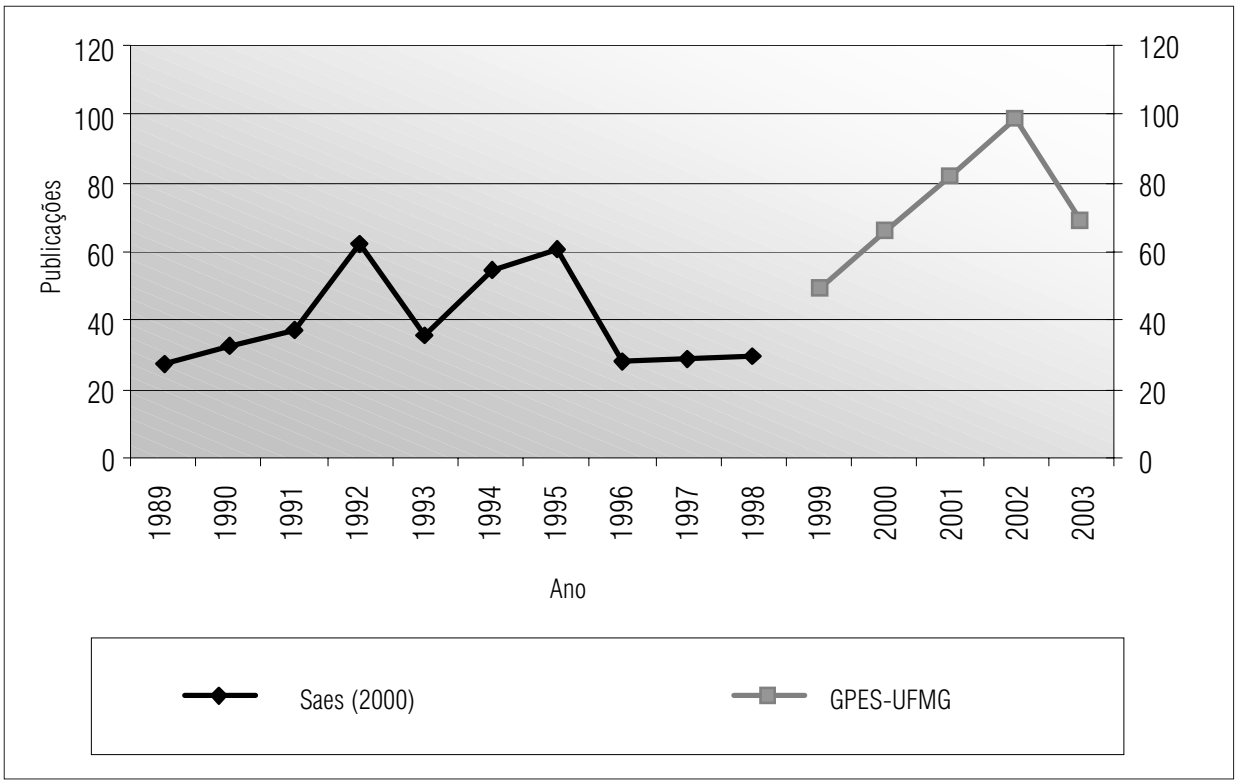

\section{Conclusões}

A análise elaborada neste artigo consistiu em um esforço de mapeamento e delimitação da economia da saúde no Brasil, permitindo a visualização de alguns aspectos importantes para a compreensão do desenvolvimento desse campo, no período entre 1999 e 2004. Aqui, adotou-se uma concepção ampliada de economia da saúde, considerando-a como um campo de fronteira entre idéias econômicas e políticas sociais, extrapolando os limites das metodologias clássicas de avaliação econômica aplicadas à saúde.

O processo de mapeamento e validação dos grupos de pesquisa cadastrados junto ao Conselho Nacional de Desenvolvimento Científico e Tecnológico (CNPq) permitiu identificar 48 grupos atuantes no campo da economia e avaliação tecnológica em saúde, com trabalhos que repercutem na área de ES. Em termos quantitativos, o conjunto é ainda pouco expressivo, representando apenas $0,3 \%$ do total de grupos de pesquisa existentes no país e $1 \%$ daqueles que atuam na área de saúde. Entretanto, a análise qualitativa desses grupos revela características bastante positivas quando se enfoca a perspectiva de consolidação da área no país. 
O primeiro aspecto digno de nota refere-se à diversidade de atuação dos grupos inventariados, situação que, a nosso ver, reflete uma grande potencialidade do campo atualmente no Brasil. Essa diversidade compreende, como atividades majoritárias, a pesquisa, as atividades ligadas a orientações de trabalhos de pós-graduação, consultoria e cursos de extensão. Além dessas, foram identificadas atividades relativas ao ensino (graduação e pós-graduação), educação permanente e cursos a distância, assessoria e cooperações técnicas, desenvolvimento e monitoramento de projetos técnicos, além da capacitação de docentes em economia e gestão em saúde.

Em relação às áreas temáticas de atuação dos grupos de pesquisa, notase uma preponderância da área de gestão em saúde, referenciada por $73 \%$ dos grupos. Esse dinamismo reflete a crescente importância da economia da saúde como suporte ao processo decisório dos gestores da saúde. A segunda principal área temática de atuação é a que enfoca medicamentos, insumos e assistência farmacêutica, seguida pela área de financiamento, alocação e eqüidade. Educação sanitária e promoção de saúde, inovação tecnológica, equipamentos médico-cirúrgicos e hospitalares também constituem áreas temáticas de atuação expressiva dos grupos pesquisados. Além dessas, foram identificadas áreas mais específicas, como mercado de saúde suplementar e gastos familiares e de empresas em saúde.

Entre os tipos de estudo desenvolvidos pelos grupos de pesquisa, constata-se um enfoque predominante na análise de custos, mas verifica-se também uma substantiva diversificação de objetos e estratégias metodológicas. Os cinco tipos de estudo mais freqüentemente desenvolvidos foram: análise de custos; eficácia, efetividade e eficiência na gestão; acessibilidade econômica; análise de custo-efetividade (58\%); e análise de custo-benefício.

A distribuição regional dos grupos de pesquisa no eixo Sudeste/Sul reproduz a concentração já identificada em outros levantamentos (Vianna, 1998; Saes, 2000). Denota-se, assim, uma maior concentração de grupos de pesquisa no estado de São Paulo, seguido por Rio de Janeiro, Minas Gerais e Rio Grande do Sul. Os restantes estão distribuídos em outros 10 estados da Federação. Essa realidade não é exclusiva da área de economia da saúde e reflete a distribuição nacional do conjunto de grupos de pesquisa.

Quando observamos a distribuição agregada por região, a maioria dos grupos concentra-se na região Sudeste (64\%), seguida pela região Nordeste (19\%) e pela região Sul (15\%). A posição relativa da região Nordeste parece refletir o esforço recente para o desenvolvimento de grupos na região, estimulado pelo projeto de Cooperação Brasil-Reino Unido para fortalecer a área de ES no país. 
Constatou-se também a existência de 82 linhas de pesquisa desenvolvidas pelos grupos com repercussão na área, reiterando o vigor, a potencialidade e a diversidade de iniciativas no campo.

Quanto ao perfil dos pesquisadores, há uma nítida predominância dos advindos da área de ciências da saúde e, nesta, uma preponderância dos profissionais graduados em medicina. A segunda área de conhecimento com maior participação é a de ciências sociais aplicadas, destacando-se os graduados em economia. O resultado mostra a progressiva inserção de duas categorias profissionais fundamentais para o desenvolvimento da área.

No que se refere à titulação acadêmica dos pesquisadores da área, ressalta-se o elevado nível de qualificação encontrado, cenário que compensa qualitativamente a ainda insuficiente quantidade de profissionais atuando nesse campo. Além disso, permite a adoção de estratégias multiplicadoras destinadas à formação de recursos humanos especializados.

O mapeamento da produção científica entre os anos 1999 e 2004 (até julho) permitiu avaliar os resumos de 2.617 trabalhos capturados nos bancos da BVS/Bireme. Foram, então, selecionados 376 trabalhos enquadrados nos critérios de classificação propostos. Desse conjunto de documentos, 64\% foram publicados como artigos em revistas e periódicos, 25\% como teses e $11 \%$ como livros.

Uma avaliação sobre o espectro de temas investigados nesses trabalhos revela a predominância (48\%) de estudos voltados para a problemática de gestão, financiamento, alocação e eqüidade, com prioridade clara (35\%) para o tema das políticas públicas e gestão em saúde. Esses achados são corroborados pelos resultados observados na análise dos grupos de pesquisa e por Vianna (1998) e Saes (2000), que identificaram que o maior interesse dos pesquisadores dessa área compreende o estudo das formas de financiamento do setor saúde, suas prioridades e a alocação de recursos. Esses autores argumentam que tal tendência pode ser explicada pelas marcantes dificuldades de financiamento e de alocação eqüitativa do Sistema Único de Saúde. Temas como medicamentos, insumos e assistência farmacêutica são objetos de $15 \%$ dos trabalhos, o mesmo ocorrendo com procedimentos clínicos e cirúrgicos. O parâmetro internacional referido por Garatini e colaboradores (2003) para a área de farmacoeconomia situa-se em torno de $35 \%$ das publicações em economia da saúde, proporção bastante superior àquela observada em nossa realidade.

Olhando o conjunto dos 376 trabalhos pelo enfoque da utilização de conceitos e ferramentas de análise relacionadas ao campo da teoria econômica, é interessante observar que praticamente $38 \%$ dos trabalhos desenvolvem alguma análise de custo e/ou de gastos em saúde. Secundariamente, estudos focados em análise de custo-efetividade, saúde suplementar, acessibilidade, 
custo de doença e demais enfoques comparecem com freqüências bem inferiores, em torno de 7\%. Essa constatação se contrapõe à observada em revisões internacionais sobre avaliações econômicas em saúde, nas quais o tipo de análise mais freqüente é a análise de custo-efetividade (CEA). Conforme recente revisão realizada, Garattini e colaboradores (2003) concluíram que, em média, 70\% dos estudos utilizam as técnicas de CEA na Itália, 62\% na Espanha e $85 \%$ na Inglaterra.

Do ponto de vista institucional, a produção científica em economia da saúde provém predominantemente dos meios acadêmicos (universidades, centros universitários, faculdades e escolas), responsáveis por $60 \%$ do total de publicações. Destacam-se a Universidade de São Paulo e a Fundação Oswaldo Cruz, com 30\% das publicações. Tal concentração reflete-se, evidentemente, no mapeamento regional, com a região Sudeste responsável por $77 \%$ das publicações identificadas. Entretanto, é relevante ressaltar a posição em segundo lugar da região Nordeste com 7\% das publicações.

Em relação à evolução da produção científica ao longo dos últimos 14 anos, a tendência é de crescimento contínuo. Para o período de 1989 a 1998, Saes (2000) identifica dois anos nos quais o volume de publicações alcança o total de 60 trabalhos/ano, enquanto no ano de 2002, atingiu-se a marca de 100 publicações/ano. O ano de 2003 apresenta uma queda de $30 \%$ de publicações em comparação com o ano de 2002, mantendo-se, porém, acima do observado nos anos 1990.

Em síntese, a análise realizada revela um cenário desafiador: de concentração regional, de saberes especializados e compartimentalizados, de sazonalidade na produção específica, num momento em que ainda é frágil a articulação entre as instituições de ensino e pesquisa e aquelas gestoras e provedoras de cuidado em saúde.

Em contraposição, importantes indicadores de potencialidades para a estruturação da área não podem ser secundarizados. A criação da Associação Brasileira de Economia da Saúde (Abres) e, mais recentemente, a instituição do Departamento de Economia da Saúde (DES), no Ministério da Saúde, podem ser consideradas marcos estruturantes do campo no Brasil. A existência de grupos de pesquisa atuantes, apresentando alta qualificação acadêmica, com relevantes contribuições à pesquisa e à formação de recursos humanos, bem como, uma crescente e comprometida produção técnico-científica devem ser considerados exemplos alvissareiros que viabilizam a formulação e implementação de políticas nesse campo.

Concluindo, espera-se que a divulgação das informações contidas neste artigo possam contribuir para uma melhor compreensão do campo da ES no Brasil, dos desafios a serem enfrentados para sua consolidação, bem como 
para motivar ações sistemáticas buscando seu fortalecimento. Esse cenário desejável propiciaria um subsídio essencial ao processo de tomada de decisão pelos gestores da saúde, que vivenciam a dificuldade cotidiana de alocar recursos escassos em um contexto de demandas crescentes. Também agregaria qualidade ao trabalho docente nesse campo do conhecimento, no fundamental ofício de educação permanente dos profissionais de saúde em nosso país. Mas, principalmente, contribuiria para que cada vez mais o nosso sistema de saúde garanta, de maneira equânime, o princípio constitucional da saúde como direito do cidadão e dever do Estado.

\section{Referências bibliográficas}

ANDRADE, E. I. G et al. Análise de situação da economia da saúde no Brasil. Perspectivas para a estruturação de um centro nacional de informações. Belo Horizonte: Coopmed, 2004.

ANNETT, H.; RIFKIN, S. Guidelines for rapid participatory appraisals to assess community health needs. A focus on health improvements for low-income urban and rural areas. Geneva: WHO/SHS/DHS, 1995.

BRASIL. Constituição da República Federativa do Brasil. Brasília: Senado Federal, 1988.

CASASNOVAS, G. L. et al. (Dir.). Los nuevos instrumentos de la gestión pública. Barcelona: La Caixa, 2003.

DEL NERO, C. R. O que é economia da saúde. In: PIOLA, S. F; VIANNA, S. M. (Orgs.). Economia da saúde: conceito e contribuição para a gestão da saúde. Brasília: Ipea, 2002.

GARATTINI, L. et al. Economic evaluations in Italy: a review of the literature. International Journal of Technology Assessment in Health Care, v. 4, p. 685-737, 2003.

GUIMARÃES, R. Bases para uma política nacional de ciência, tecnologia e inovação em saúde. Ciência \& Saúde Coletiva, v. 9, p. 375-387, 2004.

LUCCHESE, P. Editorial. Ciência \& Saúde Coletiva, Rio de Janeiro, v. 8, p. 326-327, 2003.

MEDEIROS, M. Princípios de justiça na alocação de recursos em saúde. Texto para discussão número 687. Brasília: Ipea, 1999, apud SAES, S. G. Estudo bibliométrico das publicações em economia da saúde no Brasil, 1989-1998. 2000. Dissertação (Mestrado em Administração de Serviços de Saúde) - Universidade de São Paulo, Faculdade de Saúde Pública, São Paulo, 2000.

MUSGROVE, P. Human development network: health, nutrition and population series. Washington, D.C.: The World Bank, 2004. 
SAES, S. G. Estudo bibliométrico das publicações em economia da saúde no Brasil, 19891998. 2000. Dissertação (Mestrado em Administração de Serviços de Saúde) — Universidade de São Paulo, Faculdade de Saúde Pública, São Paulo, 2000.

TAMAKI, E. M. O obstáculo da informação nos estudos em saúde. In: ENCONTRO NACIONAL DE ECONOMIA DA SAÚDE, 5., Salvador, 1999. Anais... Salvador: AbrES.

VIANNA, S. M. Evolução e perspectivas da pesquisa em economia da saúde no Brasil. Brasília: Ipea, 1998.

\section{Sites consultados}

<www.lattes.cnpq.br>. Acesso em: mar./abr. 2004.

<www.bireme.br>. Acesso em: maio/jul. 2004. 